\title{
POLYNOMIAL, RATIONAL AND ANALYTIC FIRST INTEGRALS FOR A FAMILY OF 3-DIMENSIONAL LOTKA-VOLTERRA SYSTEMS
}

\author{
JAUME LLIBRE ${ }^{1}$ AND CLÀUDIA VALLS ${ }^{2}$
}

\begin{abstract}
We extend the study of the integrability done by Leach and Miritzis (J. Nonlinear Math. Phys. 13 (2006), 535-548) on the classical model of competition between three species studied by May and Leonard (SIAM J. Appl. Math. 29 (1975), 243-256), to all real values of the parameters. Additionally our results provide all polynomial, rational and analytic first integrals of this extended model. We also classify all the invariant algebraic surfaces of these models.
\end{abstract}

\section{Introduction AND STATEMENT OF THE MAIN RESUlts}

Nonlinear ordinary differential equations appear in many branches of applied mathematics and physics. For a 3-dimensional system the existence of two first integrals whose gradients are linearly independent in $\mathbb{R}^{3}$ except perhaps in a zero Lebesgue measure set, determine completely its phase portrait because the intersections of the invariant levels of these two first integrals determine the trajectories of the system. The knowledge of a unique first integral reduces the study of the dynamics of the system from dimension 3 to dimension 2 . So the study of the existence of first integrals is an important subject in the qualitative theory of differential equations. Many different methods have been used for studying the existence of first integrals of non-linear differential systems based on: Noether symmetries [6], the Darboux theory of integrability $[8,17]$, the Lie symmetries $[1,23]$, the Painlevé analysis [3], the use of Lax pairs [12], the direct method [9, 10], the linear compatibility analysis method [24], the Carlemann embedding procedure [7, 2], the quasimonomial formalism [4], etc.

In this paper we use the Darboux theory of integrability to study the existence of first integrals for the model used by May and Leonard [18] for studying the competition among three species. This model is

$$
\begin{aligned}
& \dot{X}=X(1-X-a Y-b Z) \\
& \dot{Y}=Y(1-b X-Y-a Z) \\
& \dot{Z}=Z(1-a X-b Y-Z)
\end{aligned}
$$

2000 Mathematics Subject Classification. 34C05, 34A34, 34C14.

Key words and phrases. polynomial integrability, rational integrability, analytic integrability, Lotka-Volterra systems. 
where $a, b \in \mathbb{R}$ and the dot denotes derivative with respect to the time $t$. Note that we are interested in the integrability of system (1) for all real values of its parameters, and not only for their positive values which are the ones which biological meaning.

Doing the change of variables

$$
x=X e^{-t}, \quad y=Y e^{-t}, \quad z=Z e^{-t}, \quad s=e^{t},
$$

system (1) becomes

$$
\begin{aligned}
& x^{\prime}=-x(x+a y+b z), \\
& y^{\prime}=-y(b x+y+a z), \\
& z^{\prime}=-z(a x+b y+z),
\end{aligned}
$$

where $a, b \in \mathbb{R}$ and here the prime denotes derivative with respect to the new time $s$.

Leach and Miritzis in [13] proved that system (2) has a first integral when either $a+b=2$, or $a=b$, but in [13] it is unknown if for other values of the parameters $a$ and $b$, system (2) has or not other first integrals. As we shall show for the case $a+b=-1$, system (2) has also a first integral. Additionally we also prove that when $a=b=-1$ the system has two independent first integrals. We note that the existence of first integrals for system (2) imply the existence of invariants for system (1). Here an invariant is a first integral depending on the time.

We have computed the first integrals $H_{k}=H_{k}(x, y, z)$ when either $a+b=2$, or $a=b$ and they are

$$
\begin{array}{ll}
H_{1}=\frac{x y z}{(x+y+z)^{3}} & \text { if } a+b=2, \\
H_{2}=(x y z)^{2+a}((x-y)(x-z)(y-z))^{-(2 a+1)} & \text { if } a=b \neq 1, \\
H_{3}=\frac{x}{y}, \quad H_{4}=\frac{x}{z} & \text { if } a=b=1, \\
H_{2}, \quad H_{5}=x^{2} y^{2}-x^{2} y z-x y^{2} z+x^{2} z^{2}-x y z^{2}+y^{2} z^{2} & \text { if } a=b=-1 .
\end{array}
$$

Let $U \subset \mathbb{R}^{3}$ be an open subset. We say that the non-constant function $H: U \rightarrow$ $\mathbb{R}$ is a first integral of the polynomial vector field

$$
\mathcal{X}=-x(x+a y+b z) \frac{\partial}{\partial x}-y(b x+y+a z) \frac{\partial}{\partial y}-z(a x+b y+z) \frac{\partial}{\partial z},
$$

on $U$ associated to system (2), if $H(x(t), y(t), z(t))=$ constant for all values of $t$ for which the solution $(x(t), y(t), z(t))$ of $\mathcal{X}$ is defined on $U$. Clearly $H$ is a first integral of $\mathcal{X}$ on $U$ if and only if $\mathcal{X} H=0$ on $U$.

When $H$ is a polynomial we say that $H$ is a polynomial first integral. When $H$ is a rational function which is not a polynomial we say that $H$ is a proper rational first integral. Finally when $H$ is an analytic function we say that $H$ is an analytic first integral.

Our main results on the integrability of system (2) are the following ones.

Theorem 1. The following statements hold.

(a) The unique polynomial first integrals of system (2) are:

(a.1) $H_{2}$ when $a=b$ and either $-(2+a) /(2 a+1)$, or $-(2 a+1) /(2+a)$ is a nonnegative integer; and all the polynomial functions in the variable $\mathrm{H}_{2}$. 
(a.2) $H_{2}$ and $H_{5}$ when $a=b=-1$; and all the polynomial functions in the variables $\mathrm{H}_{2}$ and $\mathrm{H}_{5}$.

(a.3) $H_{6}=x y z$ if $a+b=-1$; and all the polynomial functions in the variable $H_{6}$. Note that $H_{6}=H_{2}$ when $a=b=-1 / 2$.

(b) The unique proper rational first integrals of system (2) are:

(b.1) $H_{3}$ and $H_{4}$ when $a=b=1$; and all proper rational functions in the variable $\mathrm{H}_{3}$ and $\mathrm{H}_{4}$.

(b.2) $H_{1}$ when $a+b=2$ and $(a, b) \neq(1,1)$; and all proper rational functions in the variable $H_{1}$.

(b.3) $H_{2}$ when $a=b$ and either $(2+a) /(2 a+1) \in \mathbb{Q}$, or $(2 a+1) /(2+a) \in \mathbb{Q}$ and $a$ does not satisfy the conditions of statement (a.1); and all proper rational functions in the variable $\mathrm{H}_{2}$.

(c) All the analytic first integrals are analytic functions in the variable $\mathrm{H}_{2}$ for the values of $a=b$ and $a$ satisfying the conditions of statement (a.1); all the analytic functions in the variables $H_{2}$ and $H_{5}$ when $a=b=-1$; and all the analytic functions in the variable $H_{6}$ when $a+b=-1$.

We remark that since the polynomial differential system (2) is homogeneous, knowing the homogeneous polynomial first integrals of the system, we can determine easily all the polynomial and analytic first integrals of system (2). For more details see Proposition 6. So statement (c) of Theorem 1 follows from statement (a) of the same theorem.

For proving Theorem 1 we shall use the invariant algebraic surfaces of system (2). This is the basis of the Darboux theory of integrability. In 1878 Darboux [8] showed how can be constructed the first integrals of planar polynomial differential systems possessing sufficient invariant algebraic curves. The Darboux theory of integrability works for real or complex polynomial ordinary differential equations. As it is explained for instance in [14] the study of complex invariant algebraic curves is necessary for obtaining all the real first integrals of a real polynomial differential equation.

The vector field $\mathcal{X}$ associated to system (2) is defined in (3). We say that $h=h(x, y, z)=0$ with $h \in \mathbb{C}[x, y, z] \backslash \mathbb{C}$ is an invariant algebraic curve of the vector field $\mathcal{X}$ if it satisfies $\mathcal{X} h=K h$ for some polynomial $K=K(x, y, z) \in \mathbb{C}[x, y, z]$, called the cofactor of $h=0$. Note that $K$ has degree at most 1 . The polynomial $h$ is called a Darboux polynomial, and we also say that $K$ is the cofactor of the Darboux polynomial $h$.

In the next result we characterize all the irreducible Darboux polynomials of system (2) with non-zero cofactor. Note that the Darboux polynomials with zero cofactor are the polynomial first integrals, which have been characterized in Theorem 1. See Proposition 3 for obtaining all the Darboux polynomials of system (2).

Theorem 2. The unique irreducible Darboux polynomials with non-zero cofactor of system (2) are:

(a) $x, y, z$ with cofactors $-(x+a y+b z),-(b x+y+a z)$ and $-(a x+b y+z)$ respectively, for all $a, b \in \mathbb{R}$.

(b) $x+y+z$ with cofactor $-(x+y+z)$ if $a+b=2$.

(c) $x-z, y-z$ and $z-x$ with cofactors $-(x+a y+z),-(a x+y+z)$ and $-(x+y+a z)$ respectively, if $b=a \neq 1$. 
(d) All homogeneous polynomials of degree 1 if $a=b=1$.

The integrability of other 3-dimensional Lotka-Volterra systems different to system (2) has already been studied. See for instance [5, 11, 15, 19, 20, 21].

In Section 2 we state and prove preliminary results for our homogeneous polynomial differential system (2). In Section 3 we prove some results of system (2) restricted to either $x=0$, or $y=0$, or $z=0$ that are important for proving Theorem 1. In section 4 we start to compute the homogeneous Darboux polynomials with non-zero cofactor. In Section 5 we start the study of the polynomial first integrals. Finally in Section 6 we prove Theorems 1 and 2.

\section{Preliminaries}

Here we provide some general results on the Darboux theory of integrability that we shall use, and some first elementary results on system (2).

Proposition 3. Let $f$ be a polynomial and $f=\prod_{j=1}^{s} f_{j}^{\alpha_{j}}$ its decomposition into irreducible factors in $\mathbb{C}[x, y, z]$. Then $f$ is a Darboux polynomial of system (2) if and only if all the $f_{j}$ are Darboux polynomials of system (2). Moreover, if $K$ and $K_{j}$ are the cofactors of $f$ and $f_{j}$ respectively, then $K=\sum_{j=1}^{s} \alpha_{j} K_{j}$.

Proof. See [14].

The following result is well-known and it is proved easily using the Darboux theory of integrability.

Proposition 4. System (2) has a proper rational first integral if and only if it has two Darboux polynomials with the same non-zero cofactor.

Lemma 5. Any Darboux polynomial $f \neq 0$ of system (2) has a cofactor of the form

$$
K=a_{1} x+a_{2} y+a_{3} z .
$$

Proof. See [22] where the authors prove Lemma 5 in a more general context of arbitrary homogeneous polynomial systems.

The following result is well-known, see for instance Proposition 1 in [16].

Proposition 6. The following statements hold:

(a) Let $f$ be a polynomial. We write $f$ in sum of its homogeneous parts as $f=f_{1}+\ldots+f_{n}$. Then $f$ is a Darboux polynomial of system (2) with cofactor $K$ if and only if $f_{j}$ is a Darboux polynomial of system (2) with cofactor $K$ for all $j=1, \ldots, n$.

(b) Let $f$ be a formal power series. We write $f$ in sum of its homogeneous parts as $f=\sum_{j \geq 1} f_{j}$, with $f_{j}$ being homogeneous polynomials of degree $j$. Then $f$ is a formal first integral of system (2) if and only if $f_{j}$ is a polynomial first integral of system (2) for all $j \geq 1$.

Proposition 7. The unique irreducible Darboux polynomials of degree 1 with nonzero cofactor of system (2) are: 
(a) $x, y, z$ with cofactors $-(x+a y+b z),-(b x+y+a z)$ and $-(a x+b y+z)$ respectively, for all $a, b \in \mathbb{R}$.

(b) $x+y+z$ with cofactor $-(x+y+z)$ if $a+b=2$.

(c) $x-z, y-z$ and $z-x$ with cofactors $-(x+a y+z),-(a x+y+z)$ and $-(x+y+a z)$ respectively, if $b=a \neq 1$.

(d) All homogeneous polynomials of degree 1 if $a=b=1$.

Proof. It follows easily from the definition of Darboux polynomial.

From statement (b.1) of Theorem 1 system (2) with $b=a=1$, or $b=a=-1$ are completely integrable (i.e. they have two first integrals whose gradients are linearly independent in $\mathbb{R}^{3}$ except perhaps in a zero Lebesgue measure set), in what follows we do not consider these particular systems.

Let $\sigma: \mathbb{C}[x, y, z] \rightarrow \mathbb{C}[x, y, z]$ be the automorphism

$$
\sigma(x)=y, \quad \sigma(y)=z, \quad \sigma(z)=x .
$$

Proposition 8. If $g$ is an irreducible homogeneous Darboux polynomial of degree $>1$ for system (2) with cofactor $K$ given by (4), then $f=g \cdot \sigma(g) \cdot \sigma^{2}(g)$ is a homogeneous Darboux polynomial of system (2) invariant by $\sigma$ with cofactor $\alpha(x+$ $y+z)$, where $\alpha=a_{1}+a_{2}+a_{3}$.

Proof. Since (2) is invariant under $\sigma$ and $\sigma^{2}$, so by Proposition $3 f=g \cdot \sigma g \cdot \sigma^{2} g$ is also a Darboux polynomial of system (2) with cofactor $K+\sigma(K)+\sigma^{2}(K)=\alpha(x+y+z)$, where $\alpha=a_{1}+a_{2}+a_{3}$.

\section{System (2) Restricted to $x=0$, or $y=0$, or $z=0$.}

We will consider in this section system (2) restricted to either $x=0$, or $y=0$, or $z=0$. Due to the invariance of system (2) with respect to $\sigma$ and $\sigma^{2}$ it is sufficient to study system (2) restricted to $z=0$, and after applying $\sigma$ and $\sigma^{2}$ to the results obtained for $z=0$ we get the results for system (2) restricted to $x=0$ and $y=0$, respectively.

Let $\mathbb{N}$ be the set of positive integers. For $n \in \mathbb{N}$ we define

(5) $C_{n}=\left\{a, b \in \mathbb{R}: a=1-n_{1}, b=1-n_{2}, n_{1} n_{2}=n, n_{1}+n_{2} \leq n, n_{1}, n_{2} \in \mathbb{N}\right\}$.

Theorem 9. For system (2) restricted to $z=0$ the following statements hold.

(a) the unique homogeneous polynomial first integrals are

$$
H=x^{1-b} y^{1-a}((b-1) x+(1-a) y)^{-1+a b}
$$

if $(a, b) \in C_{n}$ (see (5)); and all homogeneous polynomials in the variable $H$.

(b) All the irreducible Darboux polynomials with non-zero cofactor are $x$ and $y$ for all $a$ and $b$; and additionally $(b-1) x+(1-a) y$ when $a \neq 1$ and $b \neq 1$.

Proof. We consider system (2) restricted to $z=0$, i.e.,

$$
\dot{x}=-x(x+a y), \quad \dot{y}=-y(b x+y) .
$$

It follows by direct computations that $H$ as in (6) is a first integral of system (7). Furthermore, it is a homogeneous polynomial of degree $n \geq 1$ if and only if

$$
1-a=n_{1}, \quad 1-b=n_{2}, \quad a b-1=n-n_{1}-n_{2}, \quad n_{1}+n_{2} \leq n, \quad n_{1}, n_{2} \in \mathbb{N},
$$


that is if and only if

$$
1-a=n_{1}, \quad 1-b=n_{2}, \quad n_{1} n_{2}=n, \quad n_{1}+n_{2} \leq n, \quad n_{1}, n_{2} \in \mathbb{N},
$$

which clearly implies that $(a, b) \in C_{n}, n>1, n_{1} \neq 1$ and $n_{2} \neq 1$. Hence statement (a) is proved.

It also follows by direct computations that the unique irreducible Darbox polynomials of system (7) of degree one are $x$ and $y$ for all $a, b$. Moreover if $a \neq 1$ and $b \neq 1$ the polynomial $(b-1) x+(1-a) y$ is also an irreducible Darboux polynomial of sytem (7).

Let $f$ be an irreducible homogeneous Darboux polynomial of system (7) of degree $n \geq 2$. We will reach a contradiction and this will conclude the proof of the theorem. Since $f$ is a Darboux polynomial of system (7), it satisfies

$$
-x(x+a y) \frac{\partial f}{\partial x}-y(b x+y) \frac{\partial f}{\partial y}=\left(a_{1} x+a_{2} y\right) f, \quad a_{1}, a_{2} \in \mathbb{C} .
$$

We assume that either $a_{1} \neq 0$ or $a_{2} \neq 0$. If we restrict equation (23) to $x=0$ and denote by $\bar{f}$ the restriction of $f$ to $x=0$, we get that $\bar{f}=\bar{f}(y) \neq 0$ (otherwise $f$ would be reducible) is a homogeneous polynomial of degree $n$, that is $\bar{f}=\alpha_{0} y^{n}$ with $\alpha_{0} \in \mathbb{C}$. On the other hand $\bar{f}$ is a homogeneous Darboux polynomial of degree $n$ of system (7) restricted to $x=0$, that is it satisfies

$$
-y^{2} \frac{d \bar{f}}{d y}=a_{2} y \bar{f} \quad \text { i.e. } \quad \bar{f}=\alpha_{0} y^{-a_{2}}, \quad \alpha_{0} \in \mathbb{C} .
$$

Therefore equating the two expressions for $\bar{f}$ we get $a_{2}=-n$. In a similar way restricting to $y=0$ we get that $a_{1}=-n$. Thus $K=-n(x+y)$ and $(23)$ becomes

$$
-x(x+a y) \frac{\partial f}{\partial x}-y(b x+y) \frac{\partial f}{\partial y}=-n(x+y) f .
$$

Now we consider three different cases.

Case 1: $b \neq 1$ and $a \neq 1$. In this case we introduce the change of variables $(X, Y)=((b-1) x+(1-a) y, y)$. In these new variables system $(7)$ becomes

$$
X^{\prime}=\frac{X}{1-b}(X+(a+b-2) Y), \quad Y^{\prime}=\frac{Y}{1-b}(b X+(a b-1) Y) .
$$

We denote $\tilde{f}=\tilde{f}(X, Y)=f(x, y)$. We have that $\tilde{f}$ satisfies

$$
\frac{X}{1-b}(X+(a+b-2) Y) \frac{\partial \tilde{f}}{\partial X}+\frac{Y}{1-b}(b X+(a b-1) Y) \frac{\partial \tilde{f}}{\partial Y}=\frac{n}{1-b}(X+(b+a-2) Y) \tilde{f} .
$$

Now we denote by $\hat{f}$ the restriction of $\tilde{f}$ to $X=0$. Since $f$ is an irreducible polynomial we get that $\hat{f} \neq 0$ and it satisfies (10) restricted to $X=0$, that is

$$
\frac{(a b-1) Y^{2}}{1-b} \frac{d \hat{f}}{d Y}=\frac{n}{1-b}(b+a-2) Y \hat{f} .
$$

Solving this linear differential equation we deduce that $\hat{f}=\alpha_{0} y^{n(2-a-b) /(1-a b)}$, with $\alpha_{0} \in \mathbb{C}$. Since $\hat{f}$ has degree $n$, we must impose $2-a-b=1-a b$, or equivalently $(a-1)(b-1)=0$. That is $a=1$ or $b=1$, a contradiction.

Case 2: $a=1$. In this case we have $b \neq 1$. Then (9) becomes

$$
-x(x+y) \frac{\partial f}{\partial x}-y(b x+y) \frac{\partial f}{\partial y}=-n(x+y) f .
$$


Solving this partial differential equation in $f$ we get that

$$
f(x, y)=x^{n} g\left(\frac{y}{x}-\log \left(\frac{x^{b}}{y}\right)\right),
$$

where $g$ is a function in the variable $y / x-\log \left(x^{b} / y\right)$. Since $f$ must be a homogeneous polynomial of degree $n$ we must have that $g=c_{n}$ with $c_{n} \in \mathbb{C}$. Then $f(x, y)=c_{n} x^{n}$, a contradiction with the fact that $f$ is irreducible and $n>1$.

Case $3: b=1$. In this case we have $a \neq 1$. Then (9) becomes

$$
-x(x+a y) \frac{\partial f}{\partial x}-y(x+y) \frac{\partial f}{\partial y}=-n(x+y) f .
$$

From this equation we get that $x+y$ must divide $\partial f / \partial x$, and since $f$ is a homogeneous polynomial of degree $n$, we obtain that $f=f_{0}(y)+(x+y)^{2} g$, where $f_{0}(y)$ is a homogeneous polynomial in the variable $y$ of degree $n$, i.e., $f_{0}(y)=\alpha_{0} y^{n}$ with $\alpha_{0} \in \mathbb{C}$ and $g$ is a homogeneous polynomial in $x, y$ of degree $n-2$. We will show that $g=0$ and we will reach a contradiction with the fact that $f$ is irreducible of degree $n \geq 2$. We consider two subcases.

Subcase 3.1: $g$ is not divisible by $(x+y)$. Substituting $f$ in $(11)$ we obtain

$$
\begin{aligned}
& -2(x+y)\left(x^{2}+(1+a) x y+y^{2}\right) g-x(x+a y)(x+y)^{2} \frac{\partial g}{\partial x}-y(x+y)^{3} \frac{\partial g}{\partial y} \\
& =-n(x+y)^{3} g .
\end{aligned}
$$

Now simplifying by $x+y$, the above equation becomes

$$
-x(x+a y)(x+y) \frac{\partial g}{\partial x}-y(x+y)^{2} \frac{\partial g}{\partial y}=\left[2 x(x+a y)+2 y(x+y)-n(x+y)^{2}\right] g .
$$

Since $2 x(x+a y)+2 y(x+y)-n(x+y)^{2}$ is not divisible by $x+y$ we have that $g$ must be divisible by $x+y$, a contradiction.

Subcase 3.2: $g$ is divisible by $x+y$. In this case we have that $f=\alpha_{0} y^{n}+(x+y)^{m} \bar{g}$ where $m \geq 3, \alpha_{0} \in \mathbb{C}$ and $\bar{g}$ is a homogeneous polynomial in $x$ and $y$ of degree $n-m$. Furthermore, $\bar{g}$ is not divisible by $x+y$. Then $\bar{g}$ satisfies, after simplifying by $(x+y)^{m-1}$

(12) $-x(x+a y)(x+y) \frac{\partial \bar{g}}{\partial x}-y(x+y)^{2} \frac{\partial \bar{g}}{\partial y}=\left[m x(x+a y)+m y(x+y)-n(x+y)^{2}\right] \bar{g}$.

If $m=n$ then $\bar{g}$ is a constant and thus it follows from (12) that it must be the constant zero. Otherwise if $m<n$, then since $m x(x+a y)+m y(x+y)-n(x+y)^{2}$ is not divisible by $x+y$ it follows again from (12) that $\bar{g}$ must be divisible by $x+y$, a contradiction.

\section{Homogeneous Darboux polynomials With NON-ZERo COFACTOR INVARIANT BY $\sigma$}

In this section we study the homogeneous Darboux polymomials of system (2) invariants by $\sigma$ and with non-zero cofactor.

Theorem 10. System (2) has no homogeneous Darboux polynomials invariant by $\sigma$ with cofactor $K=\alpha(x+y+z), \alpha \in \mathbb{C} \backslash\{0\}$ that are irreducible and of degree $n \geq 2$. 
Proof. If we denote by $\tilde{f}=\tilde{f}(x, y)$ the restriction of $f$ to $z=0$, then $\tilde{f} \neq 0$ because $f$ is irreducible and it is a homogeneous Darboux polynomial of degree $n$ of system (7) with cofactor $\alpha(x+y)$. Then from Theorem 9 we obtain that $\tilde{f}=c x^{m_{1}} y^{m_{2}}((b-1) x+(1-a) y)^{n-m_{1}-m_{2}}$ for some integers $0 \leq m_{1}, m_{2} \leq n$ and $c \in \mathbb{C} \backslash\{0\}$. Then, the cofactor of $\tilde{f}$ is $\left((1-b) n_{2}-n\right) x+\left((1-a) n_{1}-n\right) y$. This implies that $\alpha=-n+(1-a) m_{1}$ and $(1-a) m_{1}=(1-b) m_{2}$. We consider different cases.

Case 1: $a=1, b \neq 1$. Now it follows from $(1-a) m_{1}=(1-b) m_{2}$ that $m_{2}=0$ and $\alpha=-n$. Then $\tilde{f}=c x^{n}, c \in \mathbb{C} \backslash\{0\}$. Since $f$ is invariant by $\sigma$ and $\sigma^{2}$, applying $\sigma$ and $\sigma^{2}$ to $f$ we get that $f$ can be written in the following three forms

$$
f=c x^{n}+z g=c y^{n}+x \sigma(g)=c z^{n}+y \sigma^{2}(g) .
$$

Evaluating $c x^{n}+z g=c z^{n}+y \sigma^{2}(g)$ on $z=y=0$ we get $c=0$, in contradiction with the fact that $c \neq 0$. This concludes the proof of the theorem in this case.

Case $2: b=1, a \neq 1$. It follows from $(1-a) m_{1}=(1-b) m_{2}$ and $\alpha=-n+(1-a) m_{1}$ that $m_{1}=0$ and $\alpha=-n$. Then $\tilde{f}=c y^{n}, c \in \mathbb{C} \backslash\{0\}$. Since $f$ is invariant by $\sigma$ and $\sigma^{2}$, we obtain

$$
f=c y^{n}+z g=c z^{n}+x \sigma(g)=c x^{n}+y \sigma^{2}(g) .
$$

Evaluating it on $z=y=0$ we get $c=0$, in contradiction with the fact that $c \neq 0$. Case 3: $a+b=2$ with $(a, b) \neq(1,1)$. In this case it follows from $(1-a) m_{1}=$ $(1-b) m_{2}$ that $m_{1}=m_{2}=0$. Then $\alpha=-n$ and $\tilde{f}=c(x+y)^{n}, c \in \mathbb{C} \backslash\{0\}$. On the other hand, the Darboux polynomial $f$ satisfies the equation

$-x(x+a y+(2-a) z) \frac{\partial f}{\partial x}-y((2-a) x+y+a z) \frac{\partial f}{\partial y}-z(a x+(2-a) y+z) \frac{\partial f}{\partial z}=-n(x+y+z) f$.

We introduce the change of variables $(X, Y, Z)=(x, y, z+x+y)$. In these new variables system (2) with $a+b=2$ becomes

$$
\begin{aligned}
& X^{\prime}=-X((a-1) X+2(a-1) Y+(2-a) Z), \\
& Y^{\prime}=Y(2(a-1) X+(a-1) Y-a Z), \\
& Z^{\prime}=-Z^{2} .
\end{aligned}
$$

We denote $f^{*}=f^{*}(X, Y, Z)=f(x, y, z)$. We have that $f^{*}$ satisfies

$$
\begin{aligned}
& -X((a-1) X+2(a-1) Y+(2-a) Z) \frac{\partial f^{*}}{\partial X}+Y(2(a-1) X+(a-1) Y-a Z) \frac{\partial f^{*}}{\partial Y} \\
& -Z^{2} \frac{\partial f^{*}}{\partial Z}=-n Z f^{*} .
\end{aligned}
$$

Now we denote by $\bar{f}$ the restriction of $f^{*}$ to $Z=0$. We obtain that $\bar{f}$ satisfies

$$
-(a-1) X(X+2 Y) \frac{\partial \bar{f}}{\partial X}+(a-1) Y(2 X+Y) \frac{\partial \bar{f}}{\partial Y}=0 .
$$

Since $X Y(X+Y)$ is a first integral of system (23) restricted to $Z=0$, we have that $\bar{f}=\bar{f}(X Y(X+Y))$ and it must be a homogeneous polynomial of degree $n$. Then $\bar{f}=\alpha_{0}(X Y(X+Y))^{m}$ for some $\alpha_{0} \in \mathbb{C} \backslash\{0\}, m$ a positive integer and $n=3 m$. We note that $\alpha_{0} \neq 0$ since otherwise we get a contradiction with the fact that $f$ is irreducible. Therefore

$$
f=\alpha_{0} X^{m} Y^{m}(X+Y)^{m 2}+Z g
$$


for some polynomial $g=g(X, Y, Z)$ of degree $n-1$. Imposing that $f$ is a Darboux polynomial of equation (23) with cofactor $-n Z$ we obtain, after simplifying by $Z$ that

$$
\begin{aligned}
& \alpha_{0} X^{m} Y^{m}(X+Y)^{m-1}(((a-4) m+n) X-((a+2) m-n) Y)+(n-1) Z g \\
& =X((a-1) X+2(a-1) Y+(2-a) Z) \frac{\partial g}{\partial X}-Y(2(a-1) X+(a-1) Y-a Z) \frac{\partial g}{\partial Y} \\
& +Z^{2} \frac{\partial g}{\partial Z}
\end{aligned}
$$

Setting $\bar{g}(X, Y)=g(X, Y, 0)$, we obtain that $\bar{g}$ satisfies

$$
\begin{aligned}
& \alpha_{0} X^{m} Y^{m}(X+Y)^{m-1}(((a-4) m+n) X-((a+2) m-n) Y) \\
& =X((a-1) X+2(a-1) Y) \frac{\partial \bar{g}}{\partial X}-Y(2(a-1) X+(a-1) Y) \frac{\partial \bar{g}}{\partial Y} .
\end{aligned}
$$

We note that $\bar{g}$ cannot be zero since otherwise $\alpha_{0}=0$, a contradiction. We now show that

(15) $\bar{g}=G^{m-1} h, \quad G=X Y(X+Y), h=b_{0} X^{2}+b_{1} X Y+b_{3} Y^{2}, \quad b_{0}, b_{1}, b_{2} \in \mathbb{C}$.

To prove (15) we assume that either $\bar{g}$ is not divisible by $G$, or $G$ divides $g$ but $G^{m-1}$ does not divide $\bar{g}$ and we shall arrive to contradiction.

Subcase 3.1: $\bar{g}$ is not divisible by $G$. We first denote by $g_{1}$ the restriction of $\bar{g}$ to $X=0$. Then $g_{1}$ is either 0 or a homogeneous polynomial of degree $3 m-1$ that satisfies (14) restricted to $X=0$. In this last case we have

$$
(a-1) Y^{2} \frac{d g_{1}}{d Y}=0, \quad \text { that is } g_{1}=0 .
$$

Then $\bar{g}=X h_{1}$ for some homogeneous polynomial $h_{1}=h_{1}(X, Y)$ of degree $3 m-2$.

Now we denote by $g_{2}$ the restriction of $\bar{g}$ to $Y=0$. Proceeding as for $g_{1}$ we obtain that $g_{2}=0$ and hence $\bar{g}=Y h_{2}$ for some homogeneous polynomial $h_{2}=h_{2}(X, Y)$ of degree $3 m-2$.

Finally if we denote by $g_{3}$ the restriction of $\bar{g}$ to $Y=-X$. Then $g_{3}$ is a homogeneous polynomial of degree $3 m-1$ that satisfies (14) restricted to $Y=-X$, i.e.,

$$
(1-a) X^{2} \frac{d g_{3}}{d X}=0 \quad \text { that is } g_{3}=0
$$

and $\bar{g}=(X+Y) h_{3}$ for some homogeneous polynomial $h_{3}=h_{3}(X, Y)$ of degree $3 m-2$. Therefore,

$$
\bar{g}=X Y(X+Y) h_{4}=G h_{4}
$$

for some homogeneous polynomial $h_{4}=h_{4}(X, Y, Z)$ of degree $3 m-4$, a contradiction with the fact that $\bar{g}$ is not divisible by $G$.

Subcase 3.2: $\bar{g}$ is divisible by $G$ but not by $G^{m-1}$. We have $\bar{g}=G^{l} h$ with $1 \leq l \leq$ $m-2$ and $h=h(X, Y)$ is a homogenous polynomial of degree $3(m-l)$ not divisible by $G$. We note that since $G$ is a first integral of system (23) restricted to $Z=0$, then $h$ satisfies after simplifying by $G^{l}$,

$$
\begin{aligned}
& \alpha_{0} X^{m-l} Y^{m-l}(X+Y)^{m-1-l}(((a-4) m+n) X-((a+2) m-n) Y) \\
& =(a-1) X(X+2 Y) \frac{\partial h}{\partial X}-(a-1) Y(2 X+Y) \frac{\partial h}{\partial Y} .
\end{aligned}
$$


Now proceeding for $h$ as in Case 1 we get that $h$ must be divisible by $G$, a contradiction.

Thus $\bar{g}=G^{m-1} h$ for some homogeneous polynomial $h=h(X, Y)$ of degree two that we write as $h=b_{0} X^{2}+b_{1} X Y+b_{2} Y^{2}$. This proves (15). From (15) and (16) (with $l=m-1$ ) we get, after simplifying by $G^{m-1}$ that

$$
\begin{aligned}
& \alpha_{0} X Y(((a-4) m+n) X-((a+2) m-n) Y) \\
& =(a-1) X(X+2 Y)\left(2 b_{0} X+b_{1} Y\right)-(a-1) Y(2 X+Y)\left(b_{1} X+2 b_{2} Y\right) .
\end{aligned}
$$

The unique solution of this equality is $b_{0}=b_{1}=b_{2}=0$ and $\alpha_{0}=0$, in contradiction with $\alpha_{0} \neq 0$.

Case 4: $b=a \neq \pm 1$. In this case it follows from $(1-a) m_{1}=(1-b) m_{2}$ that $m_{1}=m_{2}=m$. Then $\alpha=-n+(1-a) m$ and $\tilde{f}=c x^{m} y^{m}(x-y)^{n-2 m}, c \in \mathbb{C} \backslash\{0\}$. We first show that $\alpha=-n$. Indeed, if we denote by $f^{*}=f^{*}(z)$ the restriction of $f$ to $x=y=0$ we get that either $f^{*}=0$ or $f^{*}$ is a homogeneous polynomial of degree $n$.

In the first case we can write $f$ as $f=x f_{1}+y f_{2}$ for some homogeneous polynomials $f_{1}=f_{1}(x, y, z)$ and $f_{2}$ of degree $n-1$. Without loss of generality we can assume that $f_{2}$ does not depend on $x$. Since $f$ is invariant by $\sigma$ we get that it can be written as

$$
f=x f_{1}+y f_{2}=y \sigma\left(f_{1}\right)+z \sigma\left(f_{2}\right)=z \sigma^{2}\left(f_{1}\right)+x \sigma^{2}\left(f_{2}\right),
$$

where $f_{2}=f_{2}(y, z), \sigma\left(f_{2}\right)=\sigma\left(f_{2}\right)(z, x)$ and $\sigma^{2}\left(f_{2}\right)=\sigma^{2}\left(f_{2}\right)(x, y)$. Doing $x=y=$ 0 in $x f_{1}+y f_{2}(y, z)=y \sigma\left(f_{1}\right)+z \sigma\left(f_{2}\right)(z, x)$ we get $\sigma\left(f_{2}\right)(z, 0)=0$. Consequently $\sigma\left(f_{2}\right)(z, x)=x h(z, x)$. So $f_{2}(y, z)=z h(y, z)$. Now we have $f=x f_{1}+y z h(y, z)$. Since $\tilde{f}=f(x, y, 0)=x f_{1}(x, y, 0)=c x^{m} y^{m}(x-y)^{n-2 m}$, we get that if $m \neq 0$ then $f=c x^{m} y^{m}(x-y)^{n-2 m}+y z h(y, z)$, a contradiction with the fact that $f$ is irreducible. Then $m=0$ and from the definition of $\alpha$ we get that $\alpha=-n$.

Now we assume $f^{*} \neq 0$. Then $f^{*}$ is a homogeneous polynomial of degree $n$, i.e., $f^{*}=\alpha_{0} z^{n}$, with $\alpha_{0} \in \mathbb{C} \backslash\{0\}$. Moreover $f^{*}$ is also a Darboux polynomial of system (2) restricted to $x=y=0$ and we get

$$
-z^{2} \frac{d f^{*}}{d z}=\alpha z f^{*} \quad \text { that is } \quad f^{*}=\alpha_{0} z^{-\alpha}, \quad \alpha_{0} \in \mathbb{C},
$$

which obviously implies that $\alpha=-n$.

In short we have proved that $\alpha=-n$. Then $\tilde{f}=c(x-y)^{n}$ and $f=c(x-y)^{n}+z g$ for some homogeneous polynomial $g$ of degree $n-1$ and satisfies

$$
-x(x+a y+a z) \frac{\partial f}{\partial x}-y(a x+y+a z) \frac{\partial f}{\partial y}-z(a x+a y+z) \frac{\partial f}{\partial z}=-n(x+y+z) f .
$$

We note that in this case $x-y$ is an invariant of system (2). We introduce the change of variables $(X, Y, Z)=(x, x-y, z)$. In these new variables system $(2)$ with $b=a \neq 1$ becomes

$$
\begin{aligned}
& X^{\prime}=-X((1+a) X-a Y+a Z) \\
& Y^{\prime}=-Y(2 X-Y+a Z) \\
& Z^{\prime}=-Z(2 a X-a Y+Z) .
\end{aligned}
$$


We denote $\bar{f}=\bar{f}(X, Y, Z)=f(x, y, z)$. We have that $\bar{f}$ satisfies

$$
-X((1+a) X+a Z) \frac{\partial \bar{f}}{\partial X}-Z(2 a X+Z) \frac{\partial \bar{f}}{\partial Z}=-n(2 X+Z) \bar{f} .
$$

We note that since $f$ is irreducible we have that $\bar{f} \neq 0$. Furthermore from the discussion above we have that it is divisible by $Z$. We write it as

$\bar{f}=Z^{m} g, \quad g=\sum_{j=0}^{n-m} g_{j}(X) Z^{j}, \quad g_{j}$ is a homogeneous polynomial of degree $n-m-j$.

Then $g_{0} \neq 0$, is a homogeneous polynomial of degree $n-m$ and it follows from (18) after simplifying by $z^{m}$ and then restricting to $z=0$, that it satisfies

$$
-(1+a) X^{2} \frac{d \bar{g}_{0}}{d X}=2(a m-n) X \bar{g}_{0}, \quad \text { that is } \quad \bar{g}_{0}=\alpha_{0} X^{2(a m-n) /(a+1)}, \quad \alpha_{0} \in \mathbb{C} .
$$

Therefore since $a \neq 1$ to have that $\bar{g}_{0}$ is a polynomial of degree $n-m$ we must have $m=-n$, which is not possible. Then $\bar{g}_{0}=0$, a contradiction.

Case 5: $a \neq 1, b \neq 1, a+b \neq 2$ and $b \neq a$. In this case we have that $\alpha=$ $-n+(1-a) m_{1}$ with $(1-a) m_{1}=(1-b) m_{2}$. Then and $\tilde{f}=c x^{m_{1}} y^{m_{2}}((b-1) x+$ $(1-a) y)^{n-m_{1}-m_{2}}, c \in \mathbb{C} \backslash\{0\}$. We first show that $\alpha=-n$. Indeed, if we denote by $f^{*}=f^{*}(z)$ the restriction of $f$ to $x=y=0$ we get that either $f^{*}=0$ or $f^{*}$ is a homogeneous polynomial of degree $n$.

Assume $f^{*} \neq 0$. Proceeding as in the first paragraph of Case 4 we get that $f$ can be written as $f=x f_{1}+y z f_{2}(y, z)$ for some homogeneous polynomials $f_{1}=$ $f_{1}(x, y, z)$ and $f_{2}=f_{2}(y, z)$ of degrees $n-1$ and $n-2$, respectively. Since $\tilde{f}=$ $f(x, y, 0)=x f_{1}$, we get that if $m_{2} \neq 0$ then $f=c x^{m_{1}} y^{m_{2}}(x-y)^{n-m_{1}-m_{2}}+y z h(y, z)$, a contradiction with the fact that $f$ is irreducible. Then $m_{2}=0$ and since $a \neq 1$ we have $m_{1}=0$ and $\alpha=-n$.

Assume that $f^{*} \neq 0$. Proceeding as in the first paragraph of the proof of Case 4 we get that $\alpha=-n$.

In short $f$ satisfies

$$
-x(x+a y+b z) \frac{\partial f}{\partial x}-y(b x+y+a z) \frac{\partial f}{\partial y}-z(a x+b y+z) \frac{\partial f}{\partial z}=-n(x+y+z) f .
$$

We denote by $\tilde{f}=\tilde{f}(x, y)$ the restriction of $f$ to $z=0$. Since $\alpha=-n$ it follows from the discussion above that $\tilde{f}=c((b-1) x+(1-a) y)^{n}$ and $f=c((b-1) x+$ $(1-a) y)^{n}+z g$, where $g=g(x, y, z)$ is a homogenous polynomial of degree $n-1$. Since $f$ is invariant by $\sigma$ and $\sigma^{2}$, we obtain

$$
\begin{aligned}
f & =\alpha_{0}((b-1) x+(1-a) y)^{n}+z g=\alpha_{0}((b-1) y+(1-a) z)^{n}+x \sigma(g), \\
& =\alpha_{0}((b-1) z+(1-a) x)^{n}+y \sigma^{2}(g) .
\end{aligned}
$$

Evaluating it on $x=z=0$ we get

$$
\alpha_{0}(1-a)^{n} y^{n}=\alpha_{0}(b-1)^{n} y^{n}, \quad \text { that is }(1-a)^{n}=(b-1)^{n},
$$

in contradiction with the fact that $a+b \neq 2$ and $b \neq a$. This concludes the proof of the theorem. 


\section{Polynomial first integrals}

The main result in this section is:

Theorem 11. System (2) has no homogeneous polynomial first integrals invariant by $\sigma$ that are not divisible by a Darboux polynomial of degree one with non-zero cofactor.

Before proving Theorem 11 we show the following preliminary result.

Proposition 12. Let $n_{1}, n_{2} \in \mathbb{Z}$ with $n_{1} \neq n_{2}, n_{1}, n_{2} \geq 2$ and let $g=g(x, y)$ be $a$ homogeneous polynomial of degree $n_{1} n_{2}-1$ satisfying

$$
\begin{aligned}
& -x\left(x+\left(1-n_{1}\right) y\right) \frac{\partial g}{\partial x}-y\left(\left(1-n_{2}\right) x+y\right) \frac{\partial g}{\partial y}-\left(\left(1-n_{1}\right) x+\left(1-n_{2}\right) y\right) g \\
& +n_{1} n_{2} \alpha_{0} x^{n_{2}} y^{n_{1}}\left(-n_{2} x+n_{1} y\right)^{n_{1} n_{2}-n_{1}-n_{2}-1} F(x, y)=0,
\end{aligned}
$$

for some $\alpha_{0} \in \mathbb{C}$ with

$$
F(x, y)=\left(2 n_{2}-n_{2}^{2}-n_{1}\right) x+\left(-2 n_{1}+n_{1}^{2}+n_{2}\right) y
$$

Then $\alpha_{0}=0$.

Proof. We can assume that $\alpha_{0} \neq 0$ (otherwise there is nothing to prove) and we will arrive to a contradiction. We first show that

$$
g=\left(-n_{2} x+n_{1} y\right)^{n_{1} n_{2}-n_{1}-n_{2}-2} h_{0},
$$

with $n_{1} n_{2}-n_{1}-n_{2}-2>0$ and where $h_{0}=h_{0}(x, y)$ is a homogeneous polynomial of degree $n_{1}+n_{2}-1$. To do it we assume that either $g$ is not divisible by $-n_{2} x+n_{1} y$, or $-n_{2} x+n_{1} y$ divides $g$ but $\left(-n_{2} x+n_{1} y\right)^{n_{1} n_{2}-n_{1}-n_{2}-2}$ does not divide $g$ and we shall arrive to contradiction

We introduce the change of variables

$$
(X, Y)=\left(x,-n_{2} x+n_{1} y\right) .
$$

In these new variables system (2) restricted to $z=0$ becomes

$$
\begin{aligned}
X^{\prime} & =-\frac{X}{n_{1}}\left(\left(n_{1}+n_{2}-n_{1} n_{2}\right) X+\left(1-n_{1}\right) Y\right), \\
Y^{\prime} & =-\frac{Y}{n_{1}}\left(\left(n_{1}+n_{2}\right) X+Y\right) .
\end{aligned}
$$

We denote $g^{*}=g^{*}(X, Y)=\bar{g}(x, y)$. We have that $g^{*}$ satisfies

$$
\begin{aligned}
& -\frac{X}{n_{1}}\left(\left(n_{1}+n_{2}-n_{1} n_{2}\right) X+\left(1-n_{1}\right) Y\right) \frac{\partial g^{*}}{\partial X}-\frac{Y}{n_{1}}\left(\left(n_{1}+n_{2}\right) X+Y\right) \frac{\partial g^{*}}{\partial Y} \\
& -\left(\left(1-n_{1}\right) X+\left(1-n_{2}\right) \frac{n_{2} X+Y}{n_{1}}\right) g^{*} \\
& +n_{1} n_{2} \alpha_{0} X^{n_{2}}\left(\frac{n_{2} X+Y}{n_{1}}\right)^{n_{1}} Y^{n_{1} n_{2}-n_{1}-n_{2}-1} F^{*}(X, Y)=0,
\end{aligned}
$$

where

$$
F^{*}(X, Y)=\left(2 n_{2}-n_{2}^{2}-n_{1}\right) X+\left(-2 n_{1}+n_{1}^{2}+n_{2}\right) \frac{Y+n_{2} X}{n_{1}}
$$


Case 1: $g^{*}$ is not divisible by $Y$. Now let $\tilde{g}=\tilde{g}(X)$ be the restriction of $g^{*}$ to $Y=0$. We have that $\tilde{g} \neq 0$. Then $\tilde{g}$ is a homogeneous polynomial of degree $n_{1} n_{2}-1$ and satisfies

$$
-\frac{n_{1}+n_{2}-n_{1} n_{2}}{n_{1}} X^{2} \frac{d \tilde{g}}{d X}-\frac{X}{n_{1}}\left(n_{1}-n_{1}^{2}+n_{2}-n_{2}^{2}\right) \tilde{g}=0
$$

that is $\tilde{g}=\alpha_{2} X^{\left(n_{1}^{2}+n_{2}^{2}-n_{1}-n_{2}\right) /\left(n_{1}+n_{2}-n_{1} n_{2}\right)}$ with $\alpha_{2} \in \mathbb{C} \backslash\{0\}$. Since $\tilde{g}$ is a polynomial and $n_{1}^{2}+n_{2}^{2}-n_{1}-n_{2}>0$ and $n_{1}+n_{2}-n_{1} n_{2}<0$ we get $\tilde{g}=0$, a contradiction.

Case 2: $g^{*}$ is divisible by $Y$, but not by $Y^{n_{1} n_{2}-n_{1}-n_{2}-2}$; i.e., $g^{*}=Y^{m} g_{1}$ with $1 \leq m<n_{1} n_{2}-n_{1}-n_{2}-2$ and $g_{1}=g_{1}(X, Y)$ a homogeneous polynomial of degree $n_{1} n_{2}-2-m$ which is not divisible by $Y$. It follows from (24) that after simplifying by $Y^{m}, g_{1}$ satisfies

$$
\begin{aligned}
& -\frac{X}{n_{1}}\left(\left(n_{1}+n_{2}-n_{1} n_{2}\right) X+\left(1-n_{1}\right) Y\right) \frac{\partial g_{1}}{\partial X}-\frac{Y}{n_{1}}\left(\left(n_{1}+n_{2}\right) X+Y\right) \frac{\partial g_{1}}{\partial Y} \\
& -\left(\left(1-n_{1}+m\right) X+\left(1-n_{2}+m\right) \frac{n_{2} X+Y}{n_{1}}\right) g_{1} \\
& +n_{1} n_{2} \alpha_{0} X^{n_{2}-m}\left(\frac{n_{2} X+Y}{n_{1}}\right)^{n_{1}} Y^{n_{1} n_{2}-n_{1}-n_{2}-m-1} F^{*}(X, Y)=0 .
\end{aligned}
$$

Let $\tilde{g}_{1}$ be the restriction of $g_{1}=g_{1}(X)$ to $Y=0$, we have that $\tilde{g}_{1} \neq 0$. Then $\tilde{g}_{1}$ is a homogeneous polynomial of degree $n_{1} n_{2}-2-m$ and satisfies

(26) $-\frac{X^{2}}{n_{1}}\left(n_{1}+n_{2}-n_{1} n_{2}\right) \frac{d \tilde{g}_{1}}{d x}+\frac{X}{n_{1}}\left(n_{1}\left(n_{1}-1-m\right)+n_{2}\left(n_{2}-1-m\right)\right) \tilde{g}_{1}=0$.

Hence $\tilde{g}_{1}=\alpha_{2} x^{\left(n_{1}\left(n_{1}-1-m\right)+n_{2}\left(n_{2}-1-m\right)\right) /\left(n_{1}+n_{2}-n_{1} n_{2}\right)}$ with $\alpha_{2} \in \mathbb{C} \backslash\{0\}$. Since $\tilde{g}_{1}$ is a polynomial of degree $n_{1} n_{2}-1-m$ we must have

$$
\left(n_{1}\left(n_{1}-1-m\right)+n_{2}\left(n_{2}-1-m\right)\right)=\left(n_{1}+n_{2}-n_{1} n_{2}\right)\left(n_{1} n_{2}-m-2\right) .
$$

That is

$$
T_{n_{1}, n_{2}}=n_{1}^{2}+n_{1}-n_{1} n_{2}-m n_{1} n_{2}-n_{1}^{2} n_{2}+n_{2}^{2}+n_{2}-n_{1} n_{2}^{2}+n_{1}^{2} n_{2}^{2}=0 .
$$

Using that $m<n_{1} n_{2}-n_{1}-n_{2}-2$ we get that $0=T_{n_{1}, n_{2}}>n_{1}^{2}+n_{2}^{2}+n_{1}+n_{2}+n_{1} n_{2}>$ 0 , a contradiction. Hence (21) is proved.

It follows from (19) and (21) after simplifying by $\left(-n_{2} x+n_{1} y\right)^{n_{1} n_{2}-n_{1}-n_{2}-2}$ we get that $h_{0}$ satisfies

$$
\begin{aligned}
& -x\left(x+\left(1-n_{1}\right) y\right) \frac{\partial h_{0}}{\partial x}-y\left(\left(1-n_{2}\right) x+y\right) \frac{\partial h_{0}}{\partial y} \\
& +\left(\left(1+2 n_{1}+n_{2}-n_{1} n_{2}\right) x+\left(1+2 n_{2}+n_{1}-n_{1} n_{2}\right) y\right) h_{0} \\
& +n_{1} n_{2} \alpha_{0} x^{n_{2}} y^{n_{1}}\left(-n_{2} x+n_{1} y\right) F(x, y)=0 .
\end{aligned}
$$

We can rewrite $(27)$ in the following way

$$
\begin{aligned}
& \frac{d h_{0}}{d t}+\left(\left(1+2 n_{1}+n_{2}-n_{1} n_{2}\right) x+\left(1+2 n_{2}+n_{1}-n_{1} n_{2}\right) y\right) h_{0} \\
& +n_{1} n_{2} \alpha_{0} x^{n_{2}} y^{n_{1}}\left(-n_{2} x+n_{1} y\right) F(x, y)=0
\end{aligned}
$$

where the derivatives are evaluated along a solution of system

$$
x^{\prime}=-x\left(x+\left(1-n_{1}\right) y\right), \quad y^{\prime}=-y\left(\left(1-n_{2}\right) x+y\right) .
$$


We denote $\left(x_{n_{1}, n_{2}}(t), y_{n_{1}, n_{2}}(t)\right)$ the solution of system $(29)$ where $n_{1}, n_{2}$ are parameters. Then since (29) is invariant by the change $\tau\left(x, y, n_{1}, n_{2}\right)=\left(y, x, n_{2}, n_{1}\right)$ we have that if we denote by $h_{0, n_{1}, n_{2}}(t)=h_{0}\left(x_{n_{1}, n_{2}}(t), y_{n_{1}, n_{2}}(t)\right)$ the solution of $(28)$, then $\tau\left(h_{0, n_{1}, n_{2}}(t)\right)$ defined as $h_{0}\left(y_{n_{2}, n_{1}}(t), x_{n_{2}, n_{1}}(t)\right)$ also satisfies (28). Therefore $h_{0, n_{1}, n_{2}}(t)$ and $\tau\left(h_{0, n_{1}, n_{2}}(t)\right)$ are solutions of the same differential equation.

We shall prove that every solution $h_{0, n_{1}, n_{2}}(t)$ of system (28) is divisible by $x^{n_{2}}(t)$ (respectively $y^{n_{1}}(t)$ ). Hence since system (28) is invariant under $\tau$, we get that every solution $h_{0, n_{1}, n_{2}}(t)$ will be also divisible by $y^{n_{1}}(t)$ (respectively $x^{n_{2}}(t)$ ). The strategy will be the following. We will first show that any solution $h_{0, n_{1}, n_{2}}(t)$ of (28) must be divisible by $-n_{2} x+n_{1} y$. Then we will consider two cases: $n_{1}>n_{2}$ and $n_{1}<n_{2}$ (recall that $n_{1} \neq n_{2}$ ). In the first case we will show that $h_{0, n_{1}, n_{2}}(t)$ must be divisible by $x^{n_{2}}$ and thus by the explanation above also by $y^{n_{1}}$, and in the second case we will show that it must be divisible by $y^{n_{1}}$ and consequently by $x^{n_{2}}$. In short we will have proved that any solution $h_{0, n_{1}, n_{2}}(t)$ of $(27)$ must be of the form

and thus

$$
h_{0, n_{1}, n_{2}}(t)=c x^{n_{2}} y^{n_{1}}\left(-n_{2} x+n_{1} y\right), \quad c \in \mathbb{C},
$$

$$
g=c x^{n_{2}} y^{n_{1}}\left(-n_{2} x+n_{1} y\right)^{n_{1} n_{2}-n_{1}-n_{2}-1}, \quad c \in \mathbb{C} .
$$

Then it follows from (19) after simplifying by $x^{n_{2}} y^{n_{1}}\left(-n_{2} x+n_{1} y\right)^{n_{1} n_{2}-n_{1}-n_{2}-1}$ that

$$
\begin{aligned}
0 & =c\left(n_{1} x+n_{2} y\right)+n_{1} n_{2} \alpha_{0} F(x, y) \\
& =c\left(n_{1} x+n_{2} y\right)+n_{1} n_{2} \alpha_{0}\left(\left(2 n_{2}-n_{2}^{2}-n_{1}\right) x+\left(-2 n_{1}+n_{1}^{2}+n_{2}\right) y\right) .
\end{aligned}
$$

The unique solutions of this equation are

$$
\begin{aligned}
& c=n_{1}=0 ; \quad c=n_{2}=0 ; \quad n_{1}=n_{2}=0 ; \quad c=\alpha_{0}=0 ; \\
& c=n_{2}\left(2+\left(n_{2}-3\right) n_{2}\right), n_{1}=2-n_{2} ; \\
& c=\frac{n_{2}^{2} \alpha_{0}}{2}\left(2 n_{2}-3 \mp \sqrt{3} i\right), n_{1}=\frac{n_{2}}{2}(1 \mp \sqrt{3} i) .
\end{aligned}
$$

Note that all these solutions are in contradiction with the fact that $\alpha_{0} \neq 0$ and $n_{1}, n_{2} \geq 2$ are integers. So the proposition is proved.

To complete the proof we are left with showing first that $h_{0, n_{1}, n_{2}}(t)$ is divisible by $-n_{2} x+n_{1} y$ and $x^{n_{2}} y^{n_{1}}$ by considering the two cases mentioned above.

Now we assume that $h_{0, n_{1}, n_{2}}(t)$ is not divisible by $-n_{2} x+n_{1} y$. Introducing the variables $(X, Y)$ given in $(22)$ and proceeding as in Case 2 with $m=n_{1} n_{2}-n_{1}-$ $n_{2}-2$ and setting $h_{0}^{*}=h_{0}^{*}(X, Y)=h_{0}(x, y)$ and $\tilde{h}_{0}=\tilde{h}_{0}(X)=h_{0}^{*}(X, 0)$ we get that $\tilde{h}_{0} \neq 0$ and satisfies

$$
-\frac{X^{2}}{n_{1}}\left(n_{1}+n_{2}-n_{1} n_{2}\right) \frac{d \tilde{h}_{0}}{d x}+\frac{X}{n_{1}}\left(2 n_{1}^{2}+2 n_{2}^{2}+3 n_{1} n_{2}-n_{1}^{2} n_{2}-n_{1} n_{2}^{2}+n_{1}+n_{2}\right) \tilde{h}_{0}=0,
$$

i.e. the equation (26) with the corresponding value of $m$. Hence

$$
\tilde{h}_{0}=\alpha_{2} x^{\left(2 n_{1}^{2}+2 n_{2}^{2}+3 n_{1} n_{2}-n_{1}^{2} n_{2}-n_{1} n_{2}^{2}+n_{1}+n_{2}\right) /\left(n_{1}+n_{2}-n_{1} n_{2}\right)},
$$

with $\alpha_{2} \in \mathbb{C} \backslash\{0\}$. Since $\tilde{h}_{0}$ is a homogeneous polynomial of degree $n_{1}+n_{2}+1$ we must have

$$
2 n_{1}^{2}+2 n_{2}^{2}+3 n_{1} n_{2}-n_{1}^{2} n_{2}-n_{1} n_{2}^{2}+n_{1}+n_{2}=\left(n_{1}+n_{2}-n_{1} n_{2}\right)\left(n_{1}+n_{2}+1\right) .
$$


That is $0>T_{n_{1}, n_{2}}=\left(n_{1}+n_{2}\right)^{2}=0$, a contradiction. Hence $h_{0, n_{1}, n_{2}}(t)$ is divisible by $-n_{2} x+n_{1} y$.

Now we assume that $n_{1}>n_{2}$ and we will prove that $h_{0, n_{1}, n_{2}}(t)$ is divisible by $x^{n_{2}}$. The case $n_{1}<n_{2}$ can be studied interchanging $x$ by $y$ and $n_{1}$ by $n_{2}$.

Assume $n_{1}>n_{2}$ and we consider two cases.

Case a: $h_{0, n_{1}, n_{2}}(t)$ is not divisible by $x$. Now let $h_{1}=h_{1}(y)$ be the restriction of $h_{0, n_{1}, n_{2}}(t)$ to $x=0$. We have that $h_{1} \neq 0$. Then $h_{1}$ is a homogeneous polynomial of degree $n_{1}+n_{2}+1$ and from (27) we get

$-y^{2} \frac{d h_{1}}{d y}+\left(1+2 n_{2}+n_{1}-n_{1} n_{2}\right) y h_{1}=0 \quad$ i.e., $\quad h_{1}=\alpha_{2} y^{2 n_{2}+n_{1}+1-n_{1} n_{2}}, \quad \alpha_{2} \in \mathbb{C} \backslash\{0\}$.

Therefore $2 n_{2}+n_{1}+1-n_{1} n_{2}=n_{1}+n_{2}+1$, a contradiction with the fact that $n_{1}, n_{2} \geq 2$.

Case $b$ : $h_{0, n_{1}, n_{2}}(t)$ is divisible by $x$, but not by $x^{n_{2}}$, i.e., $h_{0, n_{1}, n_{2}}(t)=x^{m} h_{1}$ with $1 \leq m<n_{2}$ and $h_{1}=h_{1}(x, y)$ a homogeneous polynomial of degree $n_{1}+n_{2}+1-m$ that is not divisible by $x$. It follows from (27) that after simplifying by $x^{m}, h_{1}$ satisfies

$$
\begin{aligned}
& -x\left(x+\left(1-n_{1}\right) y\right) \frac{\partial h_{1}}{\partial x}-y\left(\left(1-n_{2}\right) x+y\right) \frac{\partial h_{1}}{\partial y} \\
& +\left(\left(1+2 n_{1}+n_{2}-n_{1} n_{2}-m\right) x+\left(1+2 n_{2}+n_{1}-n_{1} n_{2}-m\left(1-n_{1}\right)\right) y\right) h_{1} \\
& +n_{1} n_{2} \alpha_{0} x^{n_{2}-m} y^{n_{1}}\left(-n_{2} x+n_{1} y\right) F(x, y)=0 .
\end{aligned}
$$

Let $\bar{h}_{1}$ be the restriction of $h_{1}$ to $x=0$. Then $\bar{h}_{1}$ satisfies

$$
-y^{2} \frac{d \bar{h}_{1}}{d y}+\left(1+2 n_{2}+n_{1}-n_{1} n_{2}-m\left(1-n_{1}\right)\right) y \bar{h}_{1}=0
$$

that is

$$
\bar{h}_{1}=\alpha_{2} y^{2 n_{2}+n_{1}-n_{1} n_{2}+1-m\left(1-n_{1}\right)}, \quad \alpha_{2} \in \mathbb{C} \backslash\{0\} .
$$

Since $\bar{h}_{1}$ is a homogeneous polynomial of degree $n_{1}+n_{2}+1-m$ we must have

$$
2 n_{2}+n_{1}+1-n_{1} n_{2}-m\left(1-n_{1}\right)=n_{1}+n_{2}+1-m \text { that is } m=n_{2}-\frac{n_{2}}{n_{1}},
$$

a contradiction with the fact that $m$ is an integer and $n_{2}<n_{1}$. This completes the proof of the proposition.

Proof of Theorem 11. We proceed by contradiction. Let $f$ be a homogeneous polynomial first integral of system (2) invariant by $\sigma$ and that it is not divisible by a Darboux polynomial of degree one with non-zero cofactor of system (2). We consider three cases.

Case 1: $(a, b) \notin C_{n}$, where $C_{n}$ is defined in (5). Let $\bar{f}$ be the restriction of $f$ to $z=0$. Then $\bar{f}$ is a homogeneous polynomial and in view of Theorem 9 (see (6)) we have that $\bar{f}=0$ and thus $f=z f_{1}$ for some homogeneous polynomial $f_{1}=f_{1}(x, y, z)$, a contradiction because $z$ is a Darboux polynomial with non-zero cofactor.

Case 2: $(a, b) \in C_{n}$ and $b \neq a$. We have that $a=1-n_{1}, b=1-n_{2}$ with $n_{1} \neq n_{2}$, $n=n_{1} n_{2}$ and $n_{1}, n_{2} \geq 2$. By means of Proposition 7 and Theorem 10 we have that the unique irreducible Darboux polynomials of system (2) in this case are $x, y$ and $z$. 
We denote by $\bar{f}$ the restriction of $f$ to $z=0$. In view of Theorem 9 we have that

$$
\bar{f}=\alpha_{0} x^{n_{2}} y^{n_{1}}\left(-n_{2} x+n_{1} y\right)^{n_{1} n_{2}-n_{1}-n_{2}}, \quad \alpha_{0} \in \mathbb{C} .
$$

Note that if $\bar{f}=0$, then since $f$ is invariant by $\sigma, f$ is divisible by $x y z$ and we are done. So we can assume that $\alpha_{0} \in \mathbb{C} \backslash\{0\}$. Then

$$
f=\alpha_{0} x^{n_{2}} y^{n_{1}}\left(-n_{2} x+n_{1} y\right)^{n_{1} n_{2}-n_{1}-n_{2}}+z g,
$$

where $g=g(x, y, z)$ is a homogeneous polynomial of degree $n_{1} n_{2}-1$. Then $g$ satisfies

$$
\begin{aligned}
& -x\left(x+\left(1-n_{1}\right) y+\left(1-n_{2}\right) z\right) \frac{\partial g}{\partial x}-y\left(\left(1-n_{2}\right) x+y+\left(1-n_{1}\right) z\right) \frac{\partial g}{\partial y} \\
& -z\left(\left(1-n_{1}\right) x+\left(1-n_{2}\right) y+z\right) \frac{\partial g}{\partial z}-\left(\left(1-n_{1}\right) x+\left(1-n_{2}\right) y+z\right) g \\
& +n_{1} n_{2} \alpha_{0} x^{n_{2}} y^{n_{1}}\left(-n_{2} x+n_{1} y\right)^{n_{1} n_{2}-n_{1}-n_{2}-1} F(x, y)=0
\end{aligned}
$$

with $F(x, y)$ as in $(20)$. If we set $\bar{g}=g(x, y, 0)$, taking $z=0$ in the equation above we have

$$
\begin{aligned}
& -x\left(x+\left(1-n_{1}\right) y\right) \frac{\partial \bar{g}}{\partial x}-y\left(\left(1-n_{2}\right) x+y\right) \frac{\partial \bar{g}}{\partial y}-\left(\left(1-n_{1}\right) x+\left(1-n_{2}\right) y\right) \bar{g} \\
& \quad+n_{1} n_{2} \alpha_{0} x^{n_{2}} y^{n_{1}}\left(-n_{2} x+n_{1} y\right)^{n_{1} n_{2}-n_{1}-n_{2}-1} F(x, y)=0 .
\end{aligned}
$$

By Proposition 12 we get that $\alpha_{0}=0$, a contradiction.

Case 3: $(a, b) \in C_{n}$ and $b=a \neq \pm 1$. Then $n_{2}=n_{1}$ and $b=a=1-n_{1}$. We introduce the change of variables $(X, Y, Z)=(x, y, z-x)$. In these variables system (2) becomes

$$
\begin{aligned}
X^{\prime} & =-X\left(\left(2-n_{1}\right) X+\left(1-n_{1}\right) Y+\left(1-n_{1}\right) Z\right), \\
Y^{\prime} & =-Y\left(2\left(1-n_{1}\right) X+Y+\left(1-n_{1}\right) Z\right), \\
Z^{\prime} & =-Z\left(2 X+\left(1-n_{1}\right) Y+Z\right) .
\end{aligned}
$$

We denote $f^{*}(X, Y, Z)=f(x, y, z)$ and $\bar{f}=\bar{f}(X, Y)=f^{*}(X, Y, 0)$. Then $\bar{f}$ satisfies

$$
-X\left(\left(2-n_{1}\right) X+\left(1-n_{1}\right) Y\right) \frac{\partial \bar{f}}{\partial X}-Y\left(2\left(1-n_{1}\right) X+Y\right) \frac{\partial \bar{f}}{\partial Y}=0 .
$$

Solving it we get that $\bar{f}=\bar{f}(G)$ where

$$
G=\frac{Y^{n_{1}-2}(Y-X)^{3-2 n_{1}}}{X} .
$$

Since $G$ (or the inverse of $G$ ) must be a polynomial of degree $n \geq 1$ we must have

$$
n_{1} \leq 2, \quad n_{1} \geq \frac{3}{2} \quad \text { that is } \quad n_{1}=2 .
$$

Therefore $n_{1}=2$ and $b=a=-1$, and this case is not considered. This completes the proof of the theorem. 


\section{Proof of Theorems 1 And 2}

In this section we prove the two main results of this paper, Theorems 1 and 2 .

Proof of Theorem 1. When $a=b= \pm 1$ direct computations prove statements (a.2) and (b.1).

Let $f$ be a first integral. Now taking into account that we can always assume that the first integrals are invariant by $\sigma$ (otherwise we consider $f \cdot \sigma(f) \cdot \sigma^{2}(f)$ ), that we can restrict the polynomial first integrals to homogeneous polynomials, and that by Proposition 3 any Darboux polynomial factorizes in irreducible Darboux polynomials, by Propositions 4 and 7 and Theorem 11 any first integral (either a polynomial or a proper rational function) must be of the form

$$
f= \begin{cases}x^{m_{1}} y^{m_{2}} z^{m_{3}}(x+y+z)^{m_{4}} & \text { if } a+b=2, \\ x^{m_{1}} y^{m_{2}} z^{m_{3}}(x-y)^{m_{4}}(x-z)^{m_{5}}(y-z)^{m_{6}} & \text { if } a=b \neq \pm 1, \\ x^{m_{1}} y^{m_{2}} z^{m_{3}} & \text { for any other }(a, b) \neq \pm(1,1),\end{cases}
$$

where $m_{1}, m_{2}, m_{3}, m_{4}, m_{5}, m_{6}$ are integers. We consider three different cases.

Case 1: $a+b=2$. In this case we have that $f$ is as in (30), i.e., $f=x^{m_{1}} y^{m_{2}} z^{m_{3}}(x+$ $y+z)^{m_{4}}$ for some integers $m_{1}, m_{2}, m_{3}$ and $m_{4}$ with $m_{1}+m_{2}+m_{3}+m_{4} \neq 0$. Since $f$ is invariant by $\sigma$, we have that $f$ is equal to

$x^{m_{1}} y^{m_{2}} z^{m_{3}}(x+y+z)^{m_{4}}=y^{m_{1}} z^{m_{2}} x^{m_{3}}(x+y+z)^{m_{4}}=z^{m_{1}} x^{m_{2}} y^{m_{3}}(x+y+z)^{m_{4}}$.

Therefore equating the three relations we get

$$
m_{1}=m_{2}=m_{3}=m .
$$

Thus $f$ must be of the form $f=(x y z)^{m}(x+y+z)^{m_{4}}$. Imposing that $f$ is a first integral we get $0=-\left(m_{4}+3 m\right)(x y z)^{m}(x+y+z)^{m_{4}+1}$. Then $m_{4}=-3 m$, and thus

$$
f=\frac{x y z}{(x+y+z)^{3}} \text {. }
$$

This completes the proof of statement (b.2).

Case 2: $a=b \neq \pm 1$. In this case we have that $f$ is a polynomial or a proper rational first integral if and only if $f=x^{m_{1}} y^{m_{2}} z^{m_{3}}(x-y)^{m_{4}}(x-z)^{m_{5}}(z-y)^{m_{6}}$, for some integers $m_{1}, m_{2}, m_{3}, m_{4}, m_{5}$ and $m_{6}$. Since $f$ is invariant by $\sigma$, we have that

$$
\begin{aligned}
f & =x^{m_{1}} y^{m_{2}} z^{m_{3}}(x-y)^{m_{4}}(x-z)^{m_{5}}(z-y)^{m_{6}} \\
& =y^{m_{1}} z^{m_{2}} x^{m_{3}}(y-z)^{m_{4}}(y-x)^{m_{5}}(x-z)^{m_{6}} \\
& =z^{m_{1}} x^{m_{2}} y^{m_{3}}(z-x)^{m_{4}}(z-y)^{m_{5}}(y-x)^{m_{6}},
\end{aligned}
$$

that is

$$
m_{1}=m_{2}=m_{3}=m, \quad m_{4}=m_{5}=m_{6}=l .
$$

Thus $f$ must be of the form $f=(x y z)^{m}((x-y)(x-z)(y-z))^{l}$. Imposing that $f$ is a first integral we get

$$
0=((2 a+1) m+(2+a) l)(x y z)^{m}((x-y)(x-z)(y-z))^{l}(x+y+z) .
$$

Then $(2 a+1) m+(2+a) l=0$. If $(2 a+1) /(2+a) \notin \mathbb{Q}$ or $(2+a) /(2 a+1) \notin \mathbb{Q}$, then system (2) has no first integrals that are either a polynomial or a proper rational function. Otherwise it has a first integral of the form

$$
H(x, y, z)=(x y z)^{2+a}((x-y)(x-z)(y-z))^{-(2 a+1)} .
$$


Note that $H$ is a polynomial if and only if either $-(2+a) /(2 a+1)$, or $-(2 a+1) /(2+a)$ is a nonnegative integer. Otherwise it is a proper rational function. So statements (a.1) and (b.3) are proved.

Case 3: $a+b \neq 2, a \neq b$. In this case we have that $f=x^{m_{1}} y^{m_{2}} z^{m_{3}}$, for some integers $m_{1}, m_{2}$ and $m_{3}$. Since $f$ is invariant by $\sigma$, we have that

$$
f=x^{m_{1}} y^{m_{2}} z^{m_{3}}=y^{m_{1}} z^{m_{2}} x^{m_{3}}=z^{m_{1}} x^{m_{2}} y^{m_{3}},
$$

that is

$$
m_{1}=m_{2}=m_{3}=m \text {. }
$$

Without loss of generality we can take $m=1$. Imposing that $f$ is a first integral we get

$$
0=(1+a+b) x y z(x+y+z), \quad \text { that is } a+b=-1 .
$$

Therefore, if $a+b \neq-1$, system (2) has no first integrals that are either a polynomial or a proper rational function. If $a+b=-1$ then it has the first integral

$$
H(x, y, z)=x y z
$$

that is a polynomial. This shows statement (a.3) and concludes the proof of the theorem.

Proof of Theorem 2. If $g$ is an irreducible homogeneous Darboux polynomial of degree 1 with non-zero cofactor, then Theorem 2 follows from Proposition 7.

Now we assume that $g$ is an irreducible homogeneous Darboux polynomial of degree $n>1$ for system (2) with non-zero cofactor $K$ of the form (4). We consider two cases.

Case 1: $g$ is invariant by $\sigma$. In this case it has cofactor $K=\alpha(x+y+z)$ with $\alpha \in \mathbb{C} \backslash\{0\}$. By Theorem 10 this is not possible.

Case 2: $g$ is not invariant by $\sigma$. Then from Proposition 8 we can assume that $f=g \cdot \sigma(g) \cdot \sigma^{2}(g)$ is a homogeneous Darboux polynomial invariant by $\sigma$, with degree $3 n$ and cofactor $K=\alpha(x+y+z)$ with $\alpha \in \mathbb{C} \backslash\{0\}$. By Proposition 7 and Theorem $10 f$ has the form as in (30). We consider different subcases.

Subcase 2.1: $a+b=2$. In this case using that $f$ is invariant by $\sigma$, we get the relations of (31). Furthermore, since the degree of $f$ is $3 n$ we get that $3 m+l=3 n$, i.e., $l=3(n-m)$. Hence proceeding as in the case 1 of the proof of Theorem $1, f=$ $(x y z)^{m}(x+y+z)^{3(n-m)}$. Therefore $g=x^{m}(x+y+z)^{n-m}$, or $g=y^{m}(x+y+z)^{n-m}$, or $g=z^{m}(x+y+z)^{n-m}$, a contradiction with the fact that $g$ is irreducible of degree greater than or equal to 2 .

Subcase 2.2: $a=b \neq \pm 1$. Using that $f$ is invariant by $\sigma$, we get the relations of (32). Furthermore, since the degree of $f$ is $3 n$ we get that $l=n-m$. Hence $f=(x y z)^{m}((x-y)(x-z)(y-z))^{n-m}$. Therefore proceeding as in subcase 2.1 we get a contradiction with the fact that $g$ is irreducible of degree greater than or equal to 2 .

Subcase 2.3: $a+b \neq 2, a \neq b$. Now using that $f$ is invariant by $\sigma$, we get the relations of (33). Furthermore, since the degree of $f$ is $3 n$ we get that $m=n$. Hence $f=(x y z)^{n}$. Therefore, proceeding as in subcase 2.1 we get a contradiction with the fact that $g$ is irreducible of degree greater than or equal to 2 . 


\section{ACKNOWLEDGMENTS}

The first author has been supported by the grants MEC/FEDER MTM 200903437, CIRIT 2009SGR 410 and ICREA Academia. The second author has been partially supported by FCT through CAMGSD.

\section{REFERENCES}

[1] M.A. Almeida, M.E. Magalhães and I.C. Moreira, Lie symmetries and invariants of the Lotka-Volterra system, J. Math. Phys. 36 (1995), 1854-1867.

[2] R.F.S. Andrade And A. Rauh, The Lorenz model and the method of Carleman embedding, Phys. Lett. A 82 (1981), 276-278.

[3] T. Bountis, B. Grammaticos and A. Ramani, On the complete and partial integrability of non-Hamiltonian systems, Phys. Rep. 180 (1989) 159.

[4] L. Brenig And A. Goriely, A. Quasimonomial transformations and integrability. Partially integrable evolution equations in physics (Les Houches, 1989), 571-572, NATO Adv. Sci. Inst. Ser. C Math. Phys. Sci., 310, Kluwer Acad. Publ., Dordrecht, 1990.

[5] L. Cairó, H. Giacomini and J. Llibre, Liouvillian first integrals for the planar LotkaVolterra systems, Rendiconti del circolo matematico di Palermo 53 (2003), 389-418.

[6] F. Cantrijn and W. Sarlet, Generalizations of Noether's theorem in classical mechanics, SIAM Rev. 23 (1981), 467-494.

[7] T. Carleman, Application de la théorie des équations intégrales linéaires aux systémes d'équations différentielles non linéaires, Acta Mathematica 59 (1932), 63.

[8] G. Darboux, Mémoire sur les équations différentielles algébriques du premier ordre et du premier degré (Mélanges), Bull. Sci. math. 2ème série 2 (1878), 60-96; 123-144; 151-200.

[9] H.J. Giacomini, C.E. Repetto and O.P. Zandron, Integrals of motion of three-dimensional non-Hamiltonian dynamical systems, J. Phys. A 24 (1991), 4567-4574.

[10] J. Hietarinta, Direct methods for the search of the second invariant, Phys. Rep. 147 (1987), $87-154$.

[11] S. LABRunie, On the polynomial first integrals of the $(a, b, c)$ Lotka-Volterra system, J. Math. Phys. 37 (1996), 5539-5550.

[12] P.D. LAX, Integrals of nonlinear equations of evolution and solitary waves, Comm. Pure Appl. Math. 21 (1968), 467-490.

[13] P.G.L. Leach And J. MiRitzis, Analytic behavior of competition among three species, J. Nonlinear Math. Phys. 13 (2006), 535-548.

[14] J. LuiBRe, Integrability of polynoial differential systems, Handbook of Differential Equations, Ordinary Differential Equations, Eds. A. Cañada, P. Drabek and A. Fonda, Elsevier, 1, 2004, pp. $437-533$.

[15] J. LliBRe AND C. VAlls, Global analytic first integrals for the real planar Lotka-Volterra sysetms, J. Math. Phys. 48 (2007), 1-13.

[16] J. Llibre ANd X. Zhang, Polynomial first integrals for quasi-homogeneous polynomial differential systems, Nonlinearity 15 (2002), 1269-1280.

[17] J. Llibre and X. Zhang, Darboux theory of integrability in $C^{1}$ taking into account the mulitplicity, J. Diff. Equations 246 (2009), 541-551.

[18] R.M. May AND W.J. LEONARD, Nonlinear aspects of competition between three species, SIAM J. Appl. Math. 29 (1975), 243-256.

[19] J.M. Ollagnier, Polynomial first integrals of the Lotka-Volterra system, Bull. Sci. math. 121 (1997), 463-476.

[20] J.M. Ollagnier, Rational integration of the Lotka-Volterra system, Bull. Sci. math. 123 (1999), 437-466.

[21] J.M. OlLAGNier, Liouvillian integration of the Lotka-Volterra system, Qualitative Theory of Dynamical Systems 2 (2001), 307-358.

[22] J.M. Ollagnier, A. Nowicki And J.M. Strelcyn, On the non-existence of derivations: the proof of a theorem of Jouanolou and its developments, Bull. Sci. math. 119 (1995), 195-233.

[23] P.J. Olver, Applications of Lie groups to differential equations, Springer, New York, 1986.

[24] J.M. Strelcyn And S. Wojciechowski, A method of finding integrals for three-dimensional dynamical systems, Phys. Lett. A 133 (1988), 207-212. 
1 Departament de Matemàtiques, Universitat Autònoma de Barcelona, 08193 Bellaterra, Barcelona, Catalonia, Spain

E-mail address: jllibre@mat.uab.cat

2 Departamento de Matemática, Instituto Superior Técnico, 1049-001 Lisboa, PorTUGAL

E-mail address: cvalls@math.ist.utl.pt 